\title{
Zwischen Kontinuität und Erneuerung kritisch-materialistischer Raumforschung
}

Rezension zu Anne Vogelpohl, Boris Michel, Henrik Lebuhn, Johanna Hoerning und Bernd Belina (Hg.) (2018): Raumproduktionen II. Theoretische Kontroversen und politische Auseinandersetzungen. Münster: Verlag Westfälisches Dampfboot.

Abb. 1 Titelseite des Buches (Quelle: Verlag Westfälisches Dampfboot)

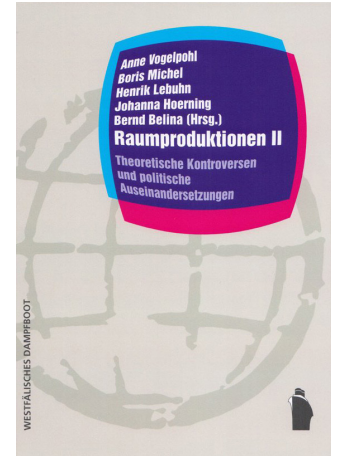

Umkämpfte und gesellschaftlich brisante Themen wie Flucht, Wohnungsnot oder Umweltbelastungen beschäftigen uns nicht nur in unserem Alltag, in Politik und Medien sowie in der Wissenschaft, sondern haben auch viel mit Raum und der Verräumlichung von Gesellschaft zu tun. Der Sammelband Raumproduktionen II. Theoretische Kontroversen und politische Auseinandersetzungen beschäftigt sich mit diesen und anderen aktuellen gesellschaftlichen Phänomenen und nimmt dabei das Verhältnis von Gesellschaft und Raum in den Blick. Mit dem Begriff der Raumproduktionen fasst der Band ein breites Spektrum an Forschungsthemen wie Migration, Mieterproteste und Landnahmen. Ausgangspunkt für die theoretische Einordnung dieser Phänomene ist ein Verständnis von Raum als soziales Produkt und als Gegenstand politischer Prozesse, die durch Auseinandersetzungen über Ungleichheiten gekennzeichnet sind.

Raumproduktionen II markiert als Jubiläumsband das zehnjährige Bestehen der gleichnamigen Reihe im Münsteraner Verlag Westfälisches Dampfboot. Der 2007 erschienene erste Band Raumproduktionen. Beiträge der Radical Geography. Eine Zwischenbilanz widmete sich noch der Übersetzung von angloamerikanischen Autoren_innen bzw. Texten aus den 1990er Jahren. Der vorliegende zweite Band versammelt hingegen acht Originalbeiträge zu aktuellen Themen der internationalen wissenschaftlichen Debatte zu Raum und Gesellschaft. Das Herausgeberteam besteht mit Bernd Belina (Frankfurt am Main) und Boris Michel (Erlangen) einerseits aus den Mitbegründern der Reihe. Andererseits bindet es mit Anne Vogelpohl (Hamburg), Henrik Lebuhn (Berlin) und Johanna Hoerning (Berlin) weitere Herausgeber_innen der deutschsprachigen kritisch-materialistischen Forschung ein.

In der Einleitung nennen Bernd Belina, Boris Michel und Anne Vogelpohl das zehnjährige Jubiläum der Reihe Raumproduktionen als Anlass und Hintergrund des Sammelbandes. Daneben setzen sie zwei Weichenstellungen für die folgenden Beiträge des Bandes. Erstens wählen die Herausgeber_innen über - wie sie es bezeichnen - „Gegenstände“ (Belina/Michel/Vogelpohl 2018: 10) als theoriegenerierende Felder einen Einstieg in die Debatte. 
Zweitens formulieren sie den Anspruch eines produktiven Dialogs von Theorieangeboten.

Mit „Gegenständen“ greifen die Beiträge erstens Themenfelder und Begrifflichkeiten aktueller theoretischer Debatten der kritischen Forschung auf. Der Zugang über die Gegenstände Infrastruktur, Körper, Umwelt, Planung und Partizipation, Protest, Urban Citizenship, Algorithmen und Big Data, Worlding und Planetarisierung eröffnet einen erfrischenden Blick auf aktuelle theoretische Diskussionen. Die Beiträge fokussieren Phänomene oder gesellschaftliche Teilbereiche, deren Auswahl aktuelle Themenkonjunkturen in der deutschsprachigen und internationalen Forschungscommunity widerspiegelt. Der Zugang über konkrete physisch-materielle, soziale oder politische Phänomene anstelle von bestimmten Theoretiker_innen oder Denkschulen[1] erlaubt eine anschauliche Argumentation basierend auf der Verknüpfung von Theorie und politischer und wissenschaftlicher Praxis. Zudem eröffnen die gegenstandsbezogenen Betrachtungen einen Zugang jenseits von disziplinären Konzepten. Gerade dieser interdisziplinäre Charakter der Debatte spiegelt sich auch in den unterschiedlichen Disziplinen der Autor_innen wider, die hier vertreten sind: Geographie, Soziologie, Architektur, Planung und Städtebau.

Der Sammelband verfolgt zweitens das Ziel, einen produktiven, pointierten Dialog zwischen kritisch-materialistischen Raumtheorien und alternativen Theorieangeboten anzuregen. In der Einleitung formulieren die Herausgeber_ innen den Anspruch, zu einer „Schärfung eines explizit kritischen und materialistisch konturierten Zugangs zu Raumproduktionen beizutragen“ (Belina/ Michel/Vogelpohl 2018: 9). Die Aufgabenstellung an die Autor_innen der Beiträge ist daher vielschichtig und durchaus anspruchsvoll gewesen: Sie sollten mit Bezug auf ihren Gegenstand durch die Abgrenzung und Auseinandersetzung mit herausfordernden theoretischen Ansätzen an einer Aktualisierung und Neuausrichtung eines kritisch-materialistischen Zugangs in der raumbezogenen Forschung mitwirken. Der Auftrag, den die Beiträge erfüllen sollen, umfasst „ein angemessenes Aufgreifen politisch brisanter Gegenstände, deren Verbindung mit einem zugespitzten Dialog zwischen verschiedenen Theorieangeboten und dabei Kritik im Sinne von Veränderbarkeit und Veränderung stetig im Blick“ (Belina/Michel/Vogelpohl 2018: 11).

Diesen gemeinsamen Arbeitsauftrag haben die Autor_innen unterschiedlich umgesetzt. Einige Beiträge - wie solche zu Infrastruktur (Stefan Höhne und Matthias Naumann), Planung und Partizipation (Nina Gribat und Manuel Lutz), Protest (Catarina Gomes de Matos und Daniel Mullis), Worlding und Planetarisierung (Johanna Hoerning) - setzen die Vorschläge der Herausgeber_innen kohärent um. Sie bieten einen systematischen Überblick über kritisch-materialistische Ansätze in ihrem Themenbereich, deren Gegenpositionen oder Erneuerungen, und schließen mit einer Synthese. Durch die dialoghafte Gegenüberstellung von Theorieangeboten in einem breit angelegten Themenbereich bieten diese Beiträge eine Übersicht zum aktuellen Stand der Forschung, wie sie im weitesten Sinne auch in ,state-of-the-art'-Artikeln in Zeitschriften zu finden sind. Dem liegt eine weite Definition des Gegenstandsbereiches zugrunde. Als Beispiel kann hier der Beitrag „Infrastruktur. Zur Analyse sozio-technischer Netzwerke zwischen altem und neuem Materialismus“ skizziert werden. Stefan Höhne und Matthias Naumann zeigen 
darin zunächst verschiedene Definitionen und disziplinäre Perspektiven auf. Im Folgenden bieten sie einen überblicksartigen Einstieg in die Debatte zum ,infrastructural turn' aus der Perspektive der Politischen Ökonomie und des ,New Materialism ' im Sinne der Actor-Network- und Assemblage-Theorie. In ihrer abschließenden Gegenüberstellung von altem und neuem Materialismus reflektieren die Autoren das ,Recht auf Infrastruktur', welches die beiden Theoriestränge verbinden und Synergien zwischen ihnen schaffen könnte.

Manche der Autor_innen wählen einen ihrem ,Gegenstand' oder dem eigenen Arbeitsbereich stärker angepassten Zugang. Der Beitrag von Nadine Marquardt und Anke Strüver „Körper. Machtgeladene Intra-aktionen zwischen Biologischem und Sozialem " bietet beispielsweise weniger eine dialoghafte Gegenüberstellung als eine breite Übersicht über theoretische Stränge der feministischen Debatte zum Körper. In einem zweiten Schritt diskutieren die Autorinnen das Verhältnis von Körper und Raum sowie die Materialität des Körpers anhand konkreter Beispiele. Henrik Lebuhn wiederum wählt einen stärker praxisbezogenen Zugang zum Gegenstandsbereich. Sein Beitrag „Urban Citizenship. Politiken der Bürgerschaft und das Recht auf Stadt“ nimmt den kommunalen Personalausweis in New York als Ausgangspunkt für eine stärker empirisch ausgerichtete Betrachtung von Stadtbürgerschaft/ Citizenship, die er in die,Recht auf Stadt'-Debatte einordnet. Maria Backhouse geht in ihrem Beitrag „Umwelt. Die neue Einhegung der Natur“ thesengeleitet vor und entwickelt David Harveys Konzept der Akkumulation der Enteignung in Bezug auf Grüne Landnahmen weiter. Dabei folgt der Beitrag einem relativ engen Begriffsverständnis des Gegenstandsbereichs Umwelt als „fortgesetzte ursprüngliche Akkumulation“ (Backhouse 2018: 61).

Diese vielseitige Zusammenstellung an Themen und die unterschiedlichen Schwerpunkte in den Beiträgen machen die Lektüre des Sammelbandes abwechslungsreich. Mit Blick auf die heterogenen Zugänge und angesichts dessen, dass die Herausgeber_innen auf eine thematische Gruppierung der Beiträge verzichtet haben, leistet das Resümee von Johanna Hoerning und Henrik Lebuhn (2018) eine Systematisierung. In ihrem abschließenden Kapitel greifen sie Ähnlichkeiten der Beiträge auf und reflektieren sie entlang von zwei Dimensionen: erstens entlang der Gegenüberstellung von Strukturen und Praktiken, zweitens entlang des Bezugs mancher Beiträge auf Henri Lefebvres Recht auf Stadt.

Im Resümee spiegelt sich jedoch auch eine Ambivalenz des Sammelbandes hinsichtlich der angestrebten Auseinandersetzung mit kritisch-materialistischen Konzepten der Raumproduktion und des Dialogs mit neueren Theorieangeboten wider. Aufbauend auf dem Eingeständnis einer heterogenen Theorielandschaft diskutieren Hoerning und Lebuhn auch die „theoretischen Fliehkräfte“ (182), die sowohl das Feld als auch den Sammelband kennzeichnen.

Die Öffnung der kritisch-materialistischen Debatte gegenüber alternativen Theorieströmungen und theoretischen Weiterentwicklungen erlaubt eine Auseinandersetzung mit konkurrierenden Ansätzen wie zum Beispiel mit dem Assemblage-Ansatz, der Akteur-Netzwerk-Theorie oder den Science and Technology Studies. Der im Sammelband geübte Dialog mit sozialkonstruktivistischen, praxis- und diskurstheoretischen Theoriesträngen erhöht damit die Anschlussfähigkeit kritisch-materialistischer Ansätze an benachbarte 
wissenschaftliche Debatten und trägt zu ihrer Weiterentwicklung bei. Im Gegenzug führt diese Öffnung der Debatte jedoch auch zu Leerstellen, die im Sammelband nicht gefüllt werden können. Gemeinsame Schlüsselautoren wie Karl Marx, David Harvey oder Henri Lefebvre werden nicht in der Vielschichtigkeit ihrer Ansätze, sondern nur punktuell in Bezug auf einzelne Konzepte aufgegriffen. Zentrale Grundbegriffe wie ,Raumproduktion' oder ,Macht, Herrschaft, Ungleichheit' werden von einzelnen Autor_innen vornehmlich in Bezug auf ihren Gegenstandsbereich erläutert. Beispielsweise verstehen Catarina Gomes de Matos und Daniel Mullis (2018) kritisch-materialistische Debatten als „Reartikulation marxistischen Denkens“ (105), welches sie im Bereich der gesellschaftsanalytischen Protestforschung vor allem um Manuel Castells und Jacques Rancière erweitert sehen (106f.). Nina Gribat und Manuel Lutz (2018) definieren dahingegen das Konzept ,kritischmaterialistisch 'in einer Fußnote (82) und üben in diesem Zusammenhang Kritik an der fehlenden Anschlussfähigkeit von (international inspirierter) kritischer Stadtforschung an die deutschsprachige Planungsdebatte (85ff.).

Beim vergleichenden Lesen der Beiträge zeigt sich, dass die Autor_innen zwar von ähnlichen Begrifflichkeiten, aber unterschiedlich nuancierten Gesellschafts- und Raumverständnissen ausgehen. Dennoch gibt es einige inhaltliche Überschneidungen zwischen den Beiträgen. Wiederholt wird auf die Wasserkrise in Flint und die urbanen Proteste in Berlin und Hamburg hingewiesen. Ein Vergleich zwischen den Ansätzen und Beiträgen hätte in der Synthese über diese gemeinsamen Beispiele noch stärker zugespitzt werden können. Zudem sind die Grenzen zwischen den ,Gegenständen“ Planung und Partizipation, Urban Citizenship und Protest und den entsprechenden Beiträgen fließend. Während der Sammelband also den Dialog nach außen, das heißt zu alternativen Theorieangeboten sucht, zeigt die Zusammenschau auch, dass eine Auseinandersetzung nach innen sowohl zwischen den Gegenstandsbereichen als auch zwischen den Strängen kritisch-materialistischer Forschung weiterhin lohnenswert ist.

Eine andere Bruchstelle des Sammelbandes bezieht sich auf die Übersetzung internationaler Debatten und eine Weiterentwicklung der deutschsprachigen Debatte. Beachtlich ist dabei, wie die Autor_innen angloamerikanische beziehungsweise internationale Beiträge auf kleinstem Raum pointiert zusammenbringen. Während dabei eine sprachliche Übersetzung ganzer Debattenfelder geleistet wird, erfolgt jedoch weniger eine inhaltliche Übertragung der stark generalisierenden Konzepte in den spezifischen Kontext. Es wird in den Beiträgen beispielsweise nicht immer deutlich, was den „eigenständige[n] deutschsprachige[n] Diskussionszusammenhang“ (Belina/Michel/Vogelpohl 2018: 9) in den jeweiligen Gegenstandsbereichen ausmacht. Eine Ausnahme davon bildet wiederum der Beitrag von Nina Gribat und Manuel Lutz „Planung und Partizipation“, der nicht nur drei unterschiedliche disziplinäre Debatten zueinander in Bezug setzt, sondern darüber hinaus auch nach dem Anschluss an die deutschsprachige Diskussion in konventionellen, das heißt auch nicht kritisch-materialistisch ausgerichteten Fachforen sowie an die deutsche Planungspraxis sucht.

Zusammenfassend lässt sich festhalten, dass der Sammelband einen durchaus lesenswerten Einstieg und Überblick in die Bandbreite der theoretischen Debatten zu Raumproduktionen im deutschsprachigen Raum 
bietet. Mit dem Fokus auf die politische Dimension der gesellschaftlichen Raumproduktionen und der Betrachtung von ,Gegenständen' bietet der Sammelband eine anschauliche Momentaufnahme der aktuellen kritischen Debatten im deutschsprachigen Wissenschaftskontext. Ob nun durch die Auseinandersetzung mit alternativen, praxis- und diskurstheoretischen sowie sozialkonstruktivistischen Theorieangeboten eine Weiterentwicklung oder Erneuerung von kritisch-materialistischen Positionen erfolgt ist, wird von den Autor_innen des Sammelbandes offengelassen. Der Jubiläumsband wirft damit offene Fragen auf, die neugierig machen darauf, wie sich die Raumproduktionen-Reihe weiterentwickeln wird. Dabei zeigt der Sammelband auch, dass die kritisch-materialistische Forschung nicht, wie von den Reihenherausgebern vormals befürchtet, an deutschsprachigen Hochschulen marginalisiert sei (Belina/Michel/Vogelpohl 2018: 8), sondern dass sie durchaus anschlussfähig an breitere disziplinäre Debatten und dialogbereit gegenüber alternativen Theorieangeboten ist.

Die Publikation dieses Beitrags wurde durch den Open-Access-Publikationsfonds der Deutschen Forschungsgemeinschaft und der Albert-LudwigsUniversität Freiburg gefördert.

\section{Endnoten}

[1] Siehe im Vergleich dazu zum Beispiel den Sammelband zu Theorien in der Raum- und Stadtforschung. Einführungen, herausgegeben von Jürgen Oßenbrügge und Anne Vogelpohl (2014).

\section{Autor innen}

Carola Fricke forscht zu Städten, Metropol- und Grenzregionen in Europa mit Fokus auf Mobilität von Politiken zwischen Ebenen und Orten sowie zur räumlichen Dimension von Politiken.

carola.fricke@geographie.uni-freiburg.de

\section{Literatur}

Backhouse, Maria (2018): Umwelt - die neue Einhegung der Natur. In: Anne Vogelpohl / Boris Michel / Henrik Lebuhn / Johanna Hoerning / Bernd Belina (Hg.): Raumproduktionen II. Theoretische Kontroversen und politische Auseinandersetzungen. Münster: Westfälisches Dampfboot, 60-80.

Belina, Bernd /Michel, Boris (Hg.) (2007): Raumproduktionen. Beiträge der Radical Geography. Eine Zwischenbilanz. (Raumproduktionen: Theorie und gesellschaftliche Praxis Band 1) Münster: Westfälisches Dampfboot.

Belina, Bernd / Michel, Boris / Vogelpohl, Anne: Gesellschaftliche Praxis im Spiegel kontroverser Raumtheorien. Zur Einleitung. In: Anne Vogelpohl / Boris Michel / Henrik Lebuhn / Johanna Hoerning / Bernd Belina (Hg.): Raumproduktionen II. Theoretische Kontroversen und politische Auseinandersetzungen. Münster: Westfälisches Dampfboot, 7-15.

de Matos, Catarina Gomes/ und Mullis, Daniel (2018): Protest - Bewegungs- und Gesellschaftsforschung aus kritisch-materialistischer Perspektive. In: Anne Vogelpohl / Boris Michel / Henrik Lebuhn / Johanna Hoerning / Bernd Belina (Hg.): Raumproduktionen II. Theoretische Kontroversen und politische Auseinandersetzungen. Münster: Westfälisches Dampfboot, 100-119. 
Gribat, Nina / Lutz, Manuel (2018): Planung und Partizipation: Zwischen Emanzipation, Kollaboration und Vereinnahmung. In: Anne Vogelpohl / Boris Michel / Henrik Lebuhn / Johanna Hoerning / Bernd Belina (Hg.): Raumproduktionen II. Theoretische Kontroversen und politische Auseinandersetzungen. Münster: Westfälisches Dampfboot, 81-99.

Hoerning, Johanna / Lebuhn, Henrik (2018): Raumproduktionen: Inspirationen aus aktuellen kritischen Debatten - ein Resümee. In: Anne Vogelpohl / Boris Michel / Henrik Lebuhn / Johanna Hoerning / Bernd Belina (Hg.): Raumproduktionen II. Theoretische Kontroversen und politische Auseinandersetzungen. Münster: Westfälisches Dampfboot, 178-188.

Oßenbrügge, Jürgen / Vogelpohl, Anne (Hg.) (2014): Theorien in der Raum- und Stadtforschung. Einführungen. Münster: Verlag Westfälisches Dampfboot.

Vogelpohl, Anne / Michel, Boris / Lebuhn, Henrik / Hoerning, Johanna / Belina, Bernd (Hg.) (2018): Raumproduktionen II. Theoretische Kontroversen und politische Auseinandersetzungen. Münster: Westfälisches Dampfboot. 